\title{
PROBING THE (EMPIRICAL) QUANTUM STRUCTURE EMBEDDED IN THE PERIODIC TABLE WITH AN EFFECTIVE BOHR MODEL
}

\author{
Wellington Nardin Favaro e Alejandro López-Castillo* \\ Departamento de Química, Universidade Federal de São Carlos, CP 676, 13560-970 São Carlos - SP, Brasil
}

Recebido em 5/3/12; aceito em 15/9/12; publicado na web em 1/2/2013

\begin{abstract}
The atomic shell structure can be observed by inspecting the experimental periodic properties of the Periodic Table. The (quantum) shell structure emerges from these properties and in this way quantum mechanics can be explicitly shown considering the (semi-) quantitative periodic properties. These periodic properties can be obtained with a simple effective Bohr model. An effective Bohr model with an effective quantum defect $(u)$ was considered as a probe in order to show the quantum structure embedded in the Periodic Table. $u(Z)$ shows a quasi-smoothed dependence of $Z$, i.e., $u(Z) \approx Z^{2 / 5}-1$.
\end{abstract}

Keywords: periodic properties; quantum structure; effective Bohr model.

\section{INTRODUCTION}

The Periodic Table (PT) is possibly the first contact that students have with Chemistry. Familiarity with the PT can help in learning important chemical concepts such as Quantum Mechanics (QM).

In general, the first introduction of QM is made theoretically by explaining the fundamental experiments or the postulates of QM. ${ }^{1}$ However, the Periodic Table (PT) could also be considered for this purpose. It is possible to easily infer the shell structure model of the atoms by simple inspection of the periodic properties (PP) of the chemical elements, e.g., the Ionization Potential (IP). If the PP can be described by simple relations, Quantum Mechanics can be introduced more naturally to chemists. The study of the PP is an active area of research and source of Chemical and Quantum insights..$^{2-9}$ Notably, the PT could be added to the list of "fundamental experimental facts".

The Schrödinger equation with spin-orbit correction is the best way to explain the quantum PP. However, an effective Bohr model should be considered for a first introduction of QM. It is possible to observe quantum manifestation on the PT empirically, probing with an effective Bohr Model. Note that the Bohr model is a limited model if used to describe the whole QM.

This work can be applied within a pedagogical approach, improving teaching and learning whereby the students must be active, creative, and participative. The effective model considered in this work is used to describe the whole PT. However, it also represents an approximate model for this. The idea of considering a model to introduce QM to students is relevant to build the thinking process of Chemistry. The teacher can use this approach to construct a challenging situation to stimulate the future learning of QM.

We intend to demonstrate that it is possible to use the PT to introduce the quantum atomic structure by inspecting the PP. The prerequisite for this process is the Bohr model of the hydrogen atom. The quasi-separability of the charges allows the use of this simple functional model. In general, why can we consider the possibility of describing many-electron atoms based on a hydrogen-like atom model, e.g., electronic distribution? This is possible since the interaction among electrons, in general, can be described by a mean field, i.e., those atoms (many-body systems) can be considered effective one-electron systems (so-called orbital model). Why does this occur? It occurs because the electrons dynamically avoid each other (namely, electrons of opposite

*e-mail: alcastil@ufscar.br spin avoid each other due to their Coulomb repulsions, and electrons of same spin avoid each other in addition, also according to the Pauli principle). In this context, one can use an effective one-electron model (such as Bohr) to describe the whole PT. Clearly it is not quantitatively good, however, it can help to simplify the introduction of the PT and quantum mechanics to students by using one simple model. The price to pay is that the simplification process can mix some distinct features. The other extreme is when one needs to describe atoms quantitatively it is better to consider the (relativistic) Schrödinger equation. It is important to emphasize that the effective Bohr model was utilized for convenience of simplicity, this model cannot substitute the Schrödinger equation for a deep comprehension of the QM.

Summarizing, we show experimental effective charge $\left(Z_{\text {eff }}\right)$ and effective quantum defect $(u)$ as a function of atomic number $(Z)$. We also show that there are several large jumps together with small ones in the $Z_{\text {eff }}(Z)$, while no large jumps occur in the experimental $u(Z)$ defined here. Due to this, we obtained a fitted simple function of $u(Z)$ allowing approximations of, e.g., $I P(Z)$ and $Z_{\text {eff }}(Z)$, both with large jumps. The main objective of this work is not to merely extend the Bohr model of the hydrogen atom, but to condense several sparse empirical complex rules into one model, which can be used to probe the quantum structure embedded in the PP of the PT. If this is reached, a simple connection between the PT with Quantum Mechanics can be obtained.

\section{RESULTS AND DISCUSSION}

\section{Effective Bohr model}

The Bohr model of the hydrogen atom describes the hydrogen-like atomic spectrum through the energy levels $E=-0.5 Z^{2} / n^{2}$, where $Z$ is the atomic number and $n$ is the principal quantum number. The two main equivalent modifications of the Bohr model usually considered to describe the energy of the polyelectronic atoms are: the effective charge $\left(Z_{e f f}\right)$ and the quantum defect $(\delta)^{1-3,10}$ or effective quantum defect $(u)$, discussed below. The correspondent energy for a neutral atom can be given by $E_{\text {Zeff }}=-0.5 Z_{\text {eff }}{ }^{2}(Z) / n^{2}(Z)$ or $E_{u}=-0.5 /$ $(n(Z)-u(Z))^{2}$, where $n(Z)$ is the principal quantum number of the ionized sub-shell valence of the atomic ground-state, as presented on the $P T . n(Z)$ is a discrete function of $Z$, which increases with atomic shell number. $u(Z)=n(Z)\left(1-1 / Z_{\text {eff }}(Z)\right)$ since $E_{\text {Zeff }}$ can be considered equivalent to $E_{u}$, i.e., $Z_{\text {eff }}{ }^{2}(Z) / n^{2}(Z)=1 /(n(Z)-u(Z))^{2}$. 
The PP can be easily described as a function of $Z$ if $Z_{e f f}(Z)$ or $u(Z)$ can be fitted as a smooth function of $Z$, i.e., without large jumps. It is possible to qualitatively (or semi-quantitatively) describe the (quasi-) $P P$ of the array of elements, which depend intrinsically on the individual atoms,${ }^{2}$ with the hydrogen-like Bohr model with an extra parameter. (The effective quantum defect also depends on the orbitals, ${ }^{2}$ consequently, a smooth function of $Z$, with only one parameter, can only describe an average behavior. This average behavior must agree qualitatively or semi-quantitatively with the experimental values.)

Below, some PPs are considered to show the quantum structure of the atoms through a simple effective model. The various PP presented in the PT are of various different natures. For example, the ionization potential $(I P)$ depends on the free neutral atom and its correspondent cation; the atomic radii are obtained from the chemical compounds. ${ }^{4}$

\section{Ionization potential}

The IP is defined as the energy difference between free cation $\left(\mathrm{A}^{+}\right)$and free neutral $\left(\mathrm{A}^{0}\right)$ atoms, both in ground-state. (Furthermore, the EA (electron affinity) is obtained from the energy difference between the free neutral $\left(\mathrm{A}^{0}\right)$ and the free anion $\left(\mathrm{A}^{-}\right)$atoms. Using the Mulliken definition, the electron-negativity $(\mathrm{EN})$ is obtained by the arithmetic average between the IP and EA $(E N \sim I P+E A)$, i.e., by the energy difference of free cation $\left(\mathrm{A}^{+}\right)$and anion $\left(\mathrm{A}^{-}\right)$atoms. The profile curves of the IP and EN are numerically similar since the IP numerical values are bigger than the EA values. Essentially it does not have a significant numerical difference if the use of IP or $E N$ are considered to obtain the average $Z_{\text {eff }}(Z)$ and $u(Z)$, i.e., $E N$ $\sim I P+E A \sim I P$.) The experimental IP as a function of $Z(I P(Z))^{11}$ is shown in Figure 1.

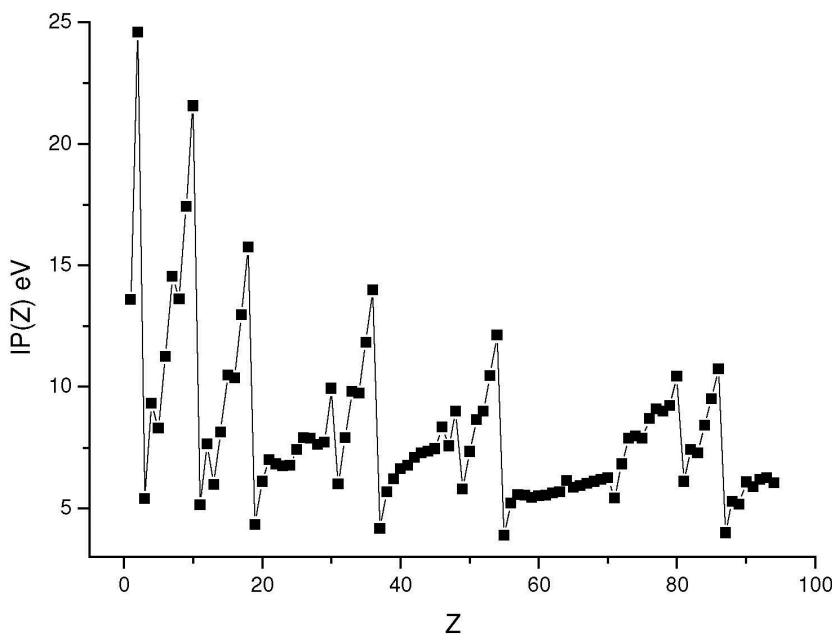

Figure 1. Experimental Ionization Potential as a function of $Z$ for the complete PT

IP for free atoms can be different from the IP for chemically bonded atoms. In spite of this, we cannot separate experimentally the IP of the bounded atom from the whole molecule. (For example; the electronic distributions for titanium ${ }^{6}$ are for: free atom or cation: $T i^{0}-[A r] 3 d^{2} 4 s^{2}, T i^{+}-[A r] 3 d^{2} 4 s^{l}$, and $T i^{2+}-[A r] 3 d^{2}$; bonded atom or cation: $T i^{0}-[A r] 3 d^{4}, T i^{+}-[A r] 3 d^{3}$, and $\left.T i^{2+}-[A r] 3 d^{2}\right)$.

That is, IP from free atoms cannot be easily correlated to fine-grained (quantitative) chemical measurements, however, the coarse-grained (semi-quantitative or qualitative) can be correlated. Simple models cannot effectively represent quantitative physical and chemical properties. However, based on the quasi separability of electrons in the atom and molecule, it is possible for the Bohr model to convey the coarse-grained physical and chemical properties. (For example, if one considers the process, e.g., $d^{2} s^{2} \rightarrow d^{2} s^{1}$ (free atom) or $d^{4} \rightarrow d^{3}$ (bonded atom) for Ti the IP error is perhaps less than the simple model approximation. In addition, the Pauling EN based on molecular aspects is similar to Mulliken EN. The chemical behavior of the elements is governed by the average atomic shell properties. But the atomic IPs, listed in the common tables, are modified by the spin-orbit coupling and sometimes by configuration changes, particularly for the transition elements. Smoother curves can be obtained if the configuration-averaged 1-electron IPs are used.

\section{The effective properties: $Z_{e f f}(Z)$ and $u(Z)$}

The $Z_{\text {eff }}$ can be defined as

$$
Z_{e f f}(Z)=n(Z)\left[I P(Z) / I P_{H}\right]^{1 / 2},
$$

obtained from the relation of $I P(Z) / I P_{H}=\left(-0.5 Z_{e f f}{ }^{2}(Z) / n^{2}(Z)\right) /(-0.5$ $Z_{H}{ }^{2} / n_{H}^{2}$ ), with $Z_{H}=n_{H}=1$ and $I P_{H}=13.6 \mathrm{eV}$ to the $\mathrm{H}$ atom. $Z_{\text {eff }}$ is an average increasing function of $Z$. The experimental $Z_{\text {eff }}(Z)$ Equation 1 is shown in Figure 2. $Z_{e f f}(Z)$ and $I P(Z)$ functions oscillate with strong dependence on the atomic family.

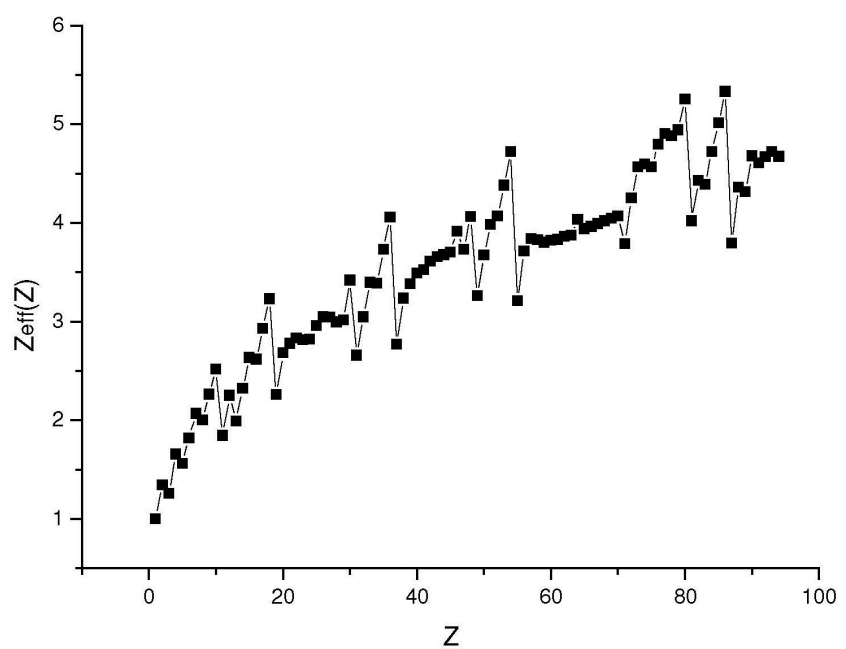

Figure 2. Experimental Effective Charge as a function of $Z$ for the complete PT

The quantum defect $\delta$ is well defined for quasi one-electron-atom, e.g., neutral alkaline-metal atoms, singly charged $C$ atoms or for an alkaline-earth cation. ${ }^{12}$ We consider that it is possible to define an effective quantum defect $u$ for the ground-state of all neutral atoms, similarly to Slater's correction. ${ }^{1}$ Slater's correction $(S)$ is defined as $S=Z-Z_{\text {eff }} \mathrm{S}$ is formulated considering that the whole system can be broken down into an effective charge and one electron (nucleus plus other electrons in the same sub-shell or otherwise). $u$ was defined for many-electron systems as an effective charge $\left(Z_{\text {eff }}\right)$. The effective quantum defect $u$ can be defined as $u=n-n^{*}$, where $n^{*}$ is the effective (principal) quantum number, $u$ is formally similar to $\delta$, but they are physically and chemically different. $u$ describes the correction in the quantum number $n$, i.e., any atom can be characterized as an effective one-electron-atom model . In particular, $u=\delta$ for alkaline atoms..$^{10,12}$ The effective quantum defect (u) cannot be mistaken with usual quantum defect $(\delta)$.

The experimental effective quantum defect $u$ can be obtained from the following equation:

$$
u(Z)=n(Z)-\left[I P_{H} / I P(Z)\right]^{1 / 2},
$$

where $u(Z=1)=0$ for the $H$ atom 


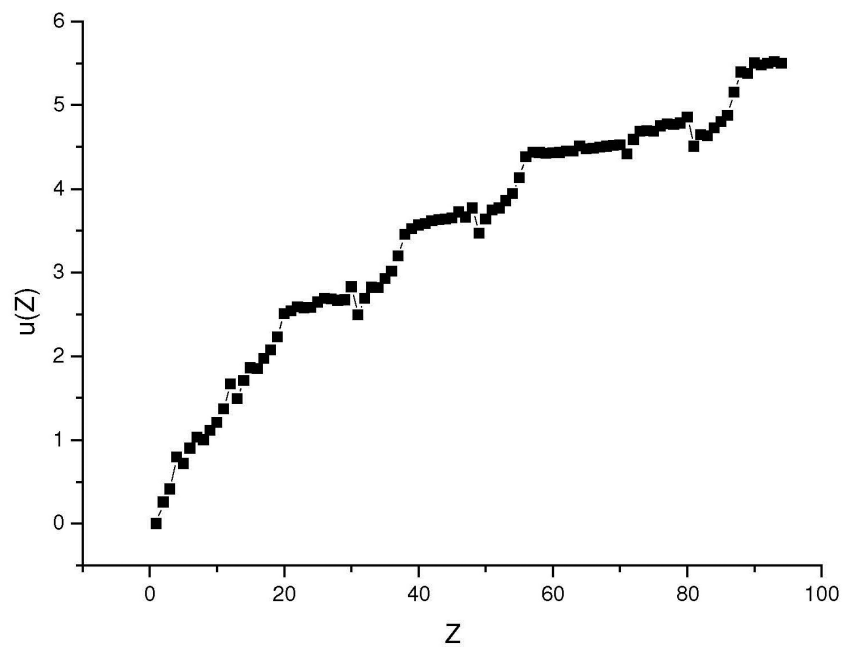

Figure 3. Experimental Effective Quantum Defect as a function of $Z$ for the complete PT

The $u(Z)$ obtained is shown in Figure 3. It is evident that $u(Z)$ has a quasi-smooth behavior as a function of $Z$. This function can be given as (see next section)

$$
\bar{u}(Z)=Z^{2 / 5}-1 .
$$

These two functions $(u(Z)$ and $\bar{u}(Z))$ are shown in Figure 4 . The function $u(Z)-\bar{u}(Z)$ was also plotted in Figure 4 and its oscillations due to the sub-shells are also evident. These oscillations can be approximately described by $u(Z)-\bar{u}(Z) \approx(1 / 5) \sin [2 \pi y(Z)]$, where $y(Z)$ $=Z / 2$ to $Z \leq 2, y(Z)=(Z-2) / 8$ to $2 \leq Z \leq 18, y(Z)=Z / 18$ to $18 \leq Z \leq 54$, and $y(Z)=(Z+10) / 32$ to $Z \geq 54$ (Figure $1 \mathrm{~S}$, supplementary material).

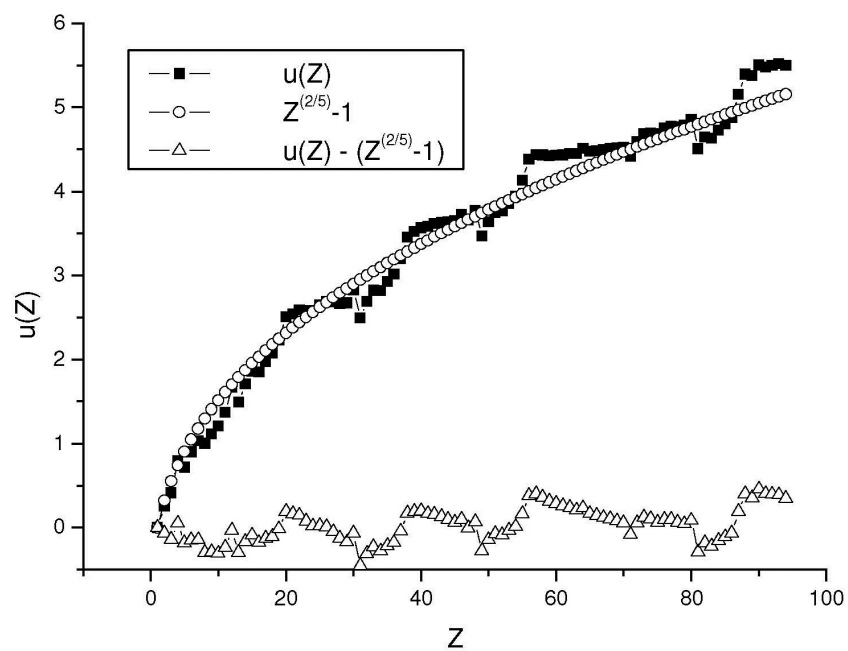

Figure 4. Experimental $u(Z)$ (square), $\bar{u}(Z)=Z^{2 / 5}-1$ (circle), and $u(Z)-\bar{u}(Z)$ (triangle) as a function of $Z$ for the complete $P T$

The increment of the effective defect (and the effective charge) increases in the order of $f, d, p, s$. Those sub-shell oscillations can also be given by the angular coefficient of $u(Z)$ as $C_{i}=d u(Z) / d Z$ for different regions of $Z$. These coefficients are remarkably distinct for $s\left(C_{s}=0.3\right), p\left(C_{p}=0.1\right), d\left(C_{d}=0.02\right)$, and $f\left(C_{f}=0.01\right)$ orbitals. For increasing numbers of valence electrons, the energy orbital order for the ground-state changes differently for the following metals: ${ }^{6}$ a) alkali : $(n+1) s<(n+1) p<n d$; b) alkali-earth : $(n+1) s<n d<(n+1)$ $p$; c) transition : $n d<(n+1) s<(n+1) p$.

The $s$ orbitals are more susceptible to change than the $d$ orbitals when there is an increase in the number of valence electrons.

The magnitude and profile of $u(Z)$ is similar to $Z_{e f f}(Z)$, but without the big jumps. Figures 2 and 3 are comparable. The similarity indicates that $u(Z)$ presents an equivalent effective chemical (and physical) meaning of the $Z_{e f f}(Z)$.

Experimental $I P(Z)$, adjusted to

$$
\overline{I P}(Z)=13.6(e V) /\left(n(Z)-Z^{2 / 5}+1\right)^{2},(4)
$$

and its best fit $\overline{I P}^{*}(Z)=13.6(\mathrm{eV}) /\left(n(Z)-\left(a Z^{b}+c\right)\right)^{2}$ (see below) curves are shown in Figure 5.

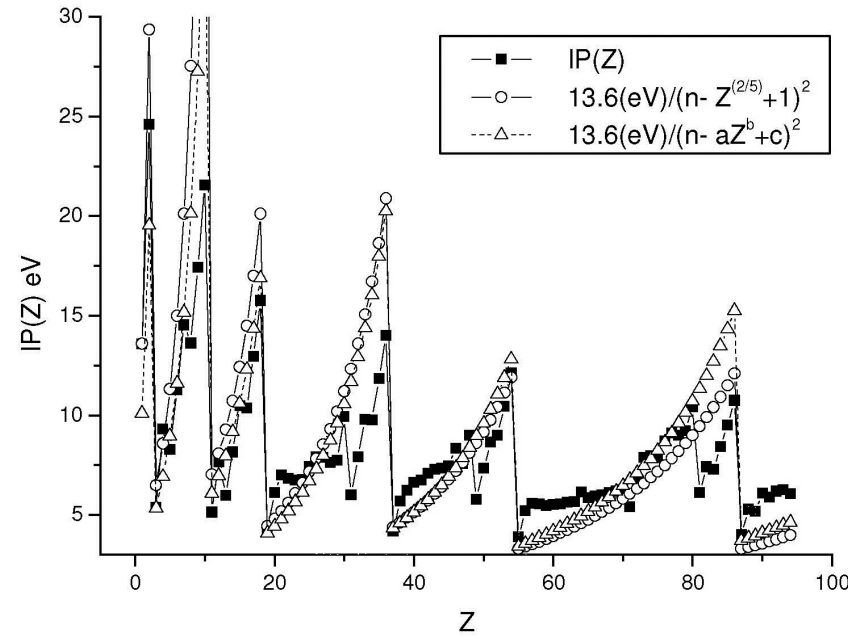

Figure 5. Experimental IP $(Z)$ (square), adjusted $\overline{I P}(Z)=13,6(\mathrm{eV}) /\left(n(Z)-Z^{2 / 5}\right.$ $+1)^{2}($ circle $)$, and $\overline{I P}(Z)=13,6(e V) /\left(n(Z)-\left(a Z^{b}+c\right)\right)^{2}$ (triangle) as a function of $Z$ for the complete $P T$

The agreement between $I P(Z)$ and $\overline{I P}(Z)($ or $\overline{I P} *(Z))$ is reasonable if only one of the parameters is considered. However, large disagreements appear in relation to noble gases, mainly for $\mathrm{Ne}$. However, it is possible to estimate the maximum and minimum value of the $I P(Z)$ by observing $\bar{u}(Z) \approx n(Z)-1$ for noble gases $(N G)$ and $\bar{u}(Z) \approx n(Z)-2$ for alkaline metals $(A M)$, which result in $\overline{I P}_{N G}(Z)=I P_{H} /(n(Z)-\bar{u}(Z))^{2}$ $\approx I P_{H}$ and $\overline{I P}_{A M}(Z)=I P_{H} /(n(Z)-\bar{u}(Z))^{2} \approx I P_{H} / 4$, respectively. The problem of determining the $Z$ dependence of the $I P$ 's in the isoelectronic series is discussed in reference 13. (The experimental Ionization Potential $(I P)$, adjusted Ionization Potential $\overline{I P}=13.6 /\left(n(Z)-Z^{2 / 5}+1\right)^{2}$, experimental Effective Charge $\left(Z_{\text {eff }}\right)$, adjusted Effective Charge $\bar{Z}_{\text {eff }}=$ $1 /\left(1+\left(1-Z^{2 / 5}\right) / n(Z)\right)$, experimental Effective Quantum Defect $(u)$, and adjusted Effective Quantum Defect $\bar{u}=Z^{2 / 5}-1$ are shown in Table $1 \mathrm{~S}$, supplementary material.)

\section{Estimative of $\bar{u}(Z)=Z^{2 / 5}-1$}

The best fit for the effective quantum defect is $\bar{u}^{*}(Z)=a Z^{b}+$ $c$, where $a=+0.98 \pm 0.16, b=+0.414 \pm 0.028$, and $c=-1.14 \pm 0.25$. However, since $u(Z=1)=0$, we also consider that $\bar{u}(Z=1)=0$, which implies that $a=-c$. We impose that $c=-a=-1$ since $a \approx 1$. We also impose that $b=p / q$, where $p$ and $q$ are small integer numbers. The approximation $b=2 / 5=0.4000$ is also a possibility. $b$ can be estimated considering simple models for the $\mathrm{He}$ atom, which is the simplest polyelectronic atom. (If $Z_{\text {eff }}$ for $H e$ can be expressed as $Z_{\text {eff }}{ }^{2} / n^{2}=$ $1 /(n-u)^{2}=1 /\left(n-Z^{\alpha}+1\right)^{2}$ we have $\alpha=\log \left(2-Z_{\text {eff }}{ }^{-1}\right) / \log (2)$ with $Z=2$ and $n=1$. For example, if $Z_{\text {eff }}=3 / 2 \rightarrow \alpha=0.415$ and $Z_{\text {eff }}=11 / 8 \rightarrow \alpha=0.348$. Two identical average effective charges $7 / 4$ are obtained considering that the $\mathrm{He}$ atom $(Z=2)$ is in a line with the nucleus at the mid-point between two electrons. $Z_{\text {eff }}=3 / 2$ is 
obtained for this line configuration, considering that after the ionization, the remnant system is represented by a hydrogen-like atom with $Z=2$, i.e., $Z_{\text {eff }}=3 / 2=2 \times 7 / 4-Z$. The average effective charges $27 / 16$ are obtained variationally for $\mathrm{He}$ atom, ${ }^{1}$ which $Z_{\text {eff }}=11 / 8=$ $2 \times 27 / 16-2$. These estimates for $b$ are only heuristic, e.g., it does not mean that the helium atom in the ground-state has a line shape). Clearly, the best average $b$ value can be obtained by solving the Schrödinger equation for all atoms. However, the simplicity would then be lost.

\section{Effective quantum number}

The effective quantum number defined as $\bar{n} *(Z)=n(Z)-\bar{u}(Z)=$ $n(Z)-Z^{2 / 5}+1$ (with only one parameter) fits the average behavior of the experimental effective quantum number $n *(Z)=n(Z) / Z_{\text {eff }}(Z)=$ $n(Z)-u(Z) . n^{*}(Z)$ and $\bar{n}^{*}(Z)$ oscillate between the effective quantum numbers of the noble gases and alkaline metals. (The principal quantum number $n(Z), n *(Z)=n(Z)-u(Z)$, and the $\bar{n}^{*}(Z)=n(Z)-Z^{2 / 5}+$ 1 are shown in Figure 2S, supplementary material).

The experimental effective quantum number $n^{*}(Z)=n(Z) / Z_{\text {eff }}(Z)$ $=n(Z)-u(Z)$ shows that the smoothed function $u(Z)$ is useful and easily interpreted, but is not discussed in the literature. The effective quantum defect $u(Z)$ can be considered a generalization of the usual quantum defect $\delta . u(Z)$ describes the correction of the quantum number $n$, i.e., any atom can be characterized as an effective one-electron-atom model.

\section{Effective charge and Slater's rules} as

The effective charge $Z_{e f f}(Z)=n(Z) /(n(Z)-u(Z))$ can be expres-

$$
\bar{Z}_{e f f}(Z)=1 /\left(1+\left(1-Z^{2 / 5}\right) / n(Z)\right) .
$$

The $Z_{\text {eff }}$ jumps are given by $n(Z)$ in the equation above since the $n(Z)$ changes when the period of the $P T$ changes. Note that the $u<$ $n$ and $\lim _{n \rightarrow \infty} \bar{Z}_{\text {eff }}=1$ for a fixed $Z$ recovers the hydrogen-like Bohr model. (The comparison between the experimental $Z_{\text {eff }}$ obtained from the $I P$ and $\bar{Z}_{e f f}(Z)$ is shown in Figure $3 \mathrm{~S}$, supplementary material).

Similarly, we can find Slater's correction $(S)^{1}$ with $Z_{\text {eff }}{ }^{*}=(Z-S)$ or $\bar{Z}_{\text {eff }}=(Z-\bar{S})$ as

$$
\bar{S}(Z)=Z-1 /\left(1+\left(1-Z^{2 / 5}\right) / n(Z)\right) .
$$

Figure 6 shows the experimental $Z_{e f f}, Z_{e f f}{ }^{*}=(Z-S)$ given by Slater's rules, ${ }^{1,14}$ along with $\bar{Z}_{\text {eff }}$ for the $1 A$ family and for the second period $(P 2)$. We can observe that $\bar{Z}_{\text {eff }}$ has a better agreement than $Z_{\text {eff }}{ }^{*}$, consequently $(Z)$ can be another alternative to Slater's rules. (The comparison between the experimental IP, IP* (obtained from Slater's rules), and $\overline{I P}$ is shown in Table $2 \mathrm{~S}$, supplementary material, and a similar agreement can be observed).

\section{Atomic radius}

The total energy is equal to half of the potential energy $E=-0.5$ $(Z / n)^{2}=0.5(-Z / r)$ for hydrogen-like atoms or for other systems with the potential given by $\sim 1 / r .{ }^{1}$ The atomic radius (in atomic unity) can be obtained by $r=n^{2} / Z$ or considering the corrections discussed above $r(Z)=n^{2}(Z) / Z_{\text {eff }}=(n(Z)-u(Z))^{2}$.

Similarly to the IP property, the ratio of the atomic radius can be given by $r(Z)=r_{H}(n(Z)-u(Z))^{2}$, where $r_{H}$ is the hydrogen's atomic radii for ground-state. If these variables are summed up, the adjusted atomic radius $\bar{r}(Z)$ is

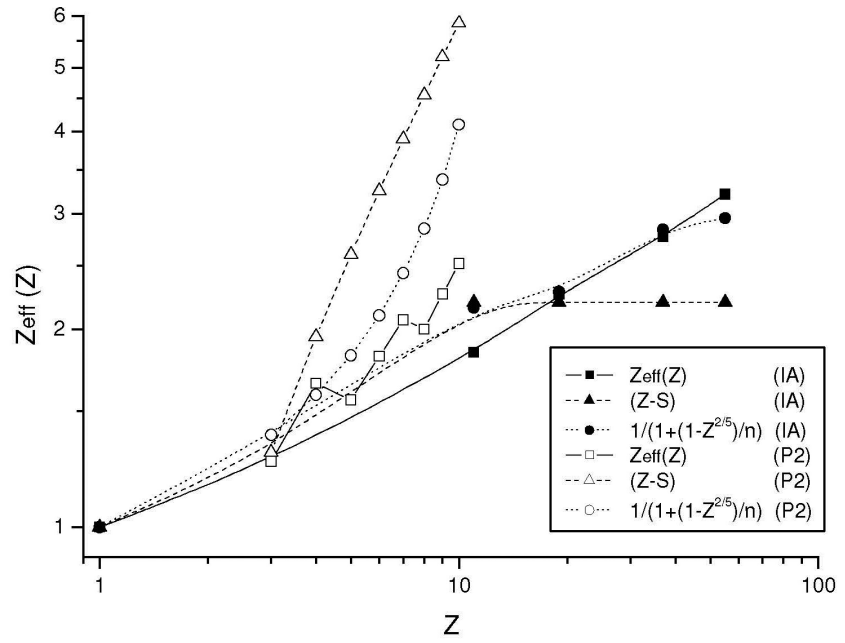

Figure 6. Experimental $Z_{\text {eff }}$ (square), $Z_{\text {eff }}{ }^{*}=(Z-S)$ given by Slater's rules (triangle) and $\bar{Z}_{\text {eff }}=1 /\left(1+\left(1-Z^{2 / 5}\right) / n(Z)\right)$ (circle) as a function of $Z$ for the $1 A$ family (solid symbols) and the second period (P2) (empty symbols)

$$
\bar{r}(Z)=r_{H}\left(n(Z)-Z^{2 / 5}+1\right)^{2} .
$$

The experimental atomic radii $r(Z)^{11}$ are shown in Figure 7 together with $\bar{r}(Z)$. A reasonable agreement between $r(Z)$ and $\bar{r}(Z)$ can be observed. Note that $r(Z)$ could depend on the definition of the $I P$.

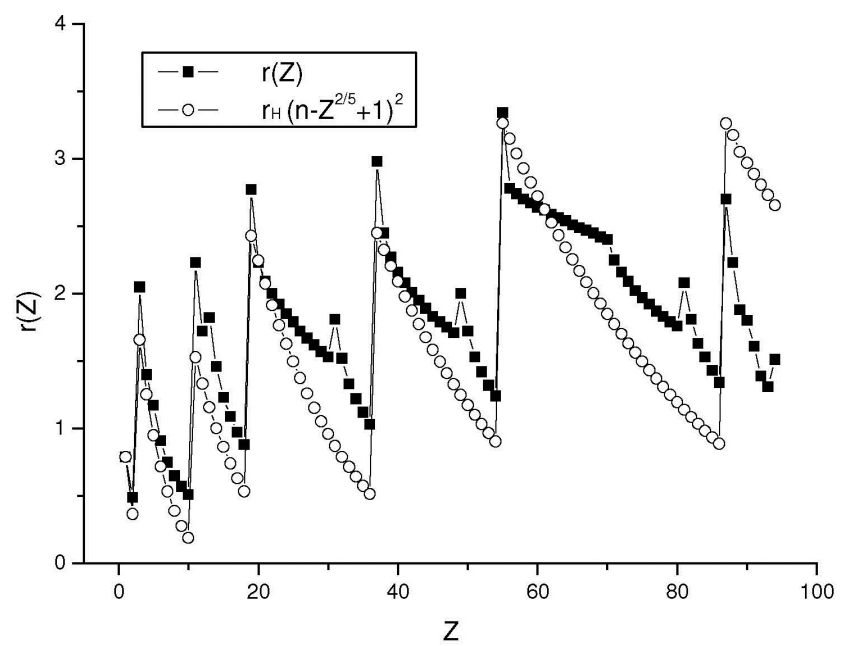

Figure 7. Experimental $r(Z)$ (square) and $\bar{r}(Z)$ (circle) as a function of $Z$ for the complete PT

Summarizing, the PPs discussed above are connected directly to the quantum atomic structure. The atomic model considered can translate this connection.

\section{CONCLUSIONS}

The (quantum) shell structure of atoms can be easily inferred by a simple inspection of the PP. Quantum Mechanics can be illustrated naturally if the PP can be described by simple relations. In other words, the empirical quantum manifestation of the PT can be probed with an effective Bohr model. Clearly, the Schrödinger equation with spin-orbit correction is the best way to explain the quantum PP and it is important to emphasize that the Bohr model is a limited model to describe the whole QM.

Some (semi-quantitative) PPs are obtained for all elements using 
the Bohr model of the hydrogen atom with the addition of a $2 / 5$ parameter. The Bohr model can describe polyelectronic atoms if the effective charge $\left(Z_{\text {eff }}(Z)\right)$ or effective quantum defect $(u(Z))$ is added. $u$ describes the correction in the $n$ quantum number, which allows any atom to be characterized as an effective one-electron-atom. The PP can be described as a function of $Z$ if $u(Z)$ can be put as a smooth (without large jumps) function of $Z \cdot u(Z)$ shows a quasi-smoothed dependence of $Z$, i.e., $u(Z) \approx Z^{2 / 5}-1$. The parameter $2 / 5$ in $u(Z)$ allows a reasonably good description of some PP's for all elements of the PT. The function $u(Z)=Z^{2 / 5}-1$ allows the obtention of simple relations for $I P(Z), Z_{e f f}(Z), \bar{S}(Z)$, and $r(Z)$. Moreover, $\bar{Z}_{e f f}=1 /\left(1+\left(1-Z^{2 / 5}\right) / n(Z)\right)$ has a better fit of the $Z_{e f f}(Z)$ than Slater's rules and permits us to also consider that the approximate ground-state energy of polyelectronic atoms can be given by $E=-0.5 /\left(n(Z)-Z^{2 / 5}+1\right)^{2}$ or after some manipulations of $E$ $=-0.5\left(\left(Z_{e f f}-1\right) / u\right)^{2}=-0.5\left(\left(Z_{\text {eff }}-1\right) /\left(Z^{2 / 5}-1\right)\right)^{2}$ for $Z>1$.

$u(Z)$ presents an equivalent effective meaning of $Z_{e f f}(Z)$ for effective models. An effective model can describe the real atoms and the PP better than sparse empirical rules. Several empirical rules alone cannot have a simple interpretation. However, a simple general construction, such as the Bohr model, provides a simple rationalization to understand the more general scenario of the PT for example. The Bohr model with the 2/5 parameter has that prerogative. If one can understand the PT, Quantum Mechanics emerges from its inspection.

Quantum Mechanics can be effectively illustrated for chemistry students by referring to a model fit for the atomic shell structure. PPs are derived from the quantum atomic structure while the term "periodic" is a direct consequence of the "quantum structure".

\section{SUPPLEMENTARY MATERIAL}

The supplementary material, available at http://quimicanova. sbq.org.br, with free access, in pdf file, contains figures, which show the periodic behaviors of the $u(Z), n(Z), n *(Z), *(Z)$, and eff $^{\text {There }}$ are two tables with the experimental and fitted values of the $\operatorname{IP}(Z)$, $Z_{\text {eff }}(Z)$, and $u(Z)$.

\section{ACKNOWLEDGMENTS}

A. López-Castillo acknowledges Prof. W. H. E. Schwarz for evaluable discussions. This work is supported by FAPESP/Brazil (2010/11385-2).

\section{REFERENCES}

1. Levine, I. N.; Quantum Chemistry, Prentice Hall: Englewood Cliffs, $4^{\text {th }}$ ed.; 1991.

2. Schwarz, W. H. E.; Found. Chem. 2007, 9, 139.

3. Wang, S.-G.; Schwarz, W. H. E.; Angew. Chem., Int. Ed. 2009, 48, 3404.

4. Schwarz, W. H. E.; Wang, S.-G.; Int. J. Quantum Chem. 2010, 110, 1455 .

5. Schwarz, W. H. E.; Rich, R. L.; J. Chem. Educ. 2010, 87, 435.

6. Schwarz, W. H. E.; J. Chem. Educ. 2010, 87, 444.

7. Scerri, E. R.; Int. J. Quantum Chem. 2009, 109, 959.

8. Ostrovsky, V. N.; Ann. N. Y. Acad. Sci. 2003, 988, 182.

9. Tolentino, M.; Rocha-Filho, R. C.; Chagas, A. P.; Quim. Nova 1997, 20, 103.

10. Sala, O.; Quim. Nova 2007, 30, 2057.

11. http://www.chemglobe.org/ptoe/download/index.php, accessed April 2011.

12. Foot, C. J.; Atomic Physics, Oxford Master Series in Physics, Oxford University Press: New York, 2005.

13. Agmon, N.; J. Chem. Educ. 1988, 65, 42.

14. Duarte, H. A.; Química Nova na Escola 2003, nº 17, 22. 
PROBING THE (EMPIRICAL) QUANTUM STRUCTURE EMBEDDED IN THE PERIODIC TABLE WITH AN EFFECTIVE BOHR MODEL

Wellington Nardin Favaro e Alejandro López-Castillo*

Departamento de Química, Universidade Federal de São Carlos, CP 676, 13560-970 São Carlos - SP, Brasil

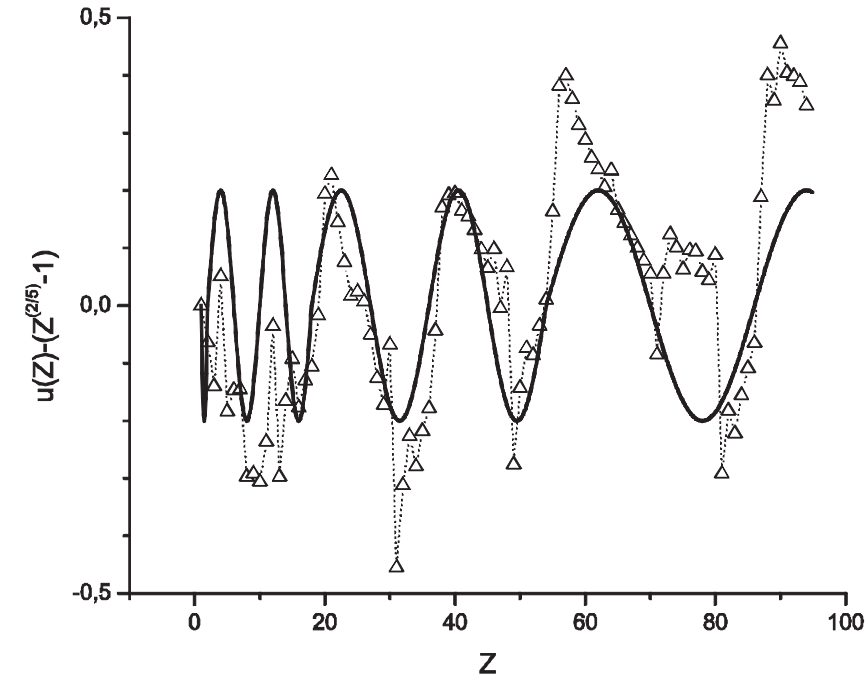

Figure 1S. $-u(Z)-\bar{u}(Z) \approx(1 / 5) \sin [2 \pi y(Z)]$ (solid line), where $y(Z)=Z / 2$ to $Z \leq 2, y(Z)=(Z-2) / 8$ to $2 \leq Z \leq 18, y(Z)=Z / 18$ to $18 \leq Z \leq 54$, and $y(Z)=$ $(Z+10) / 32$ to $Z \geq 54 ; u(Z)-\bar{u}(Z)$ (triangle) as function of $Z$ for complete $P T$

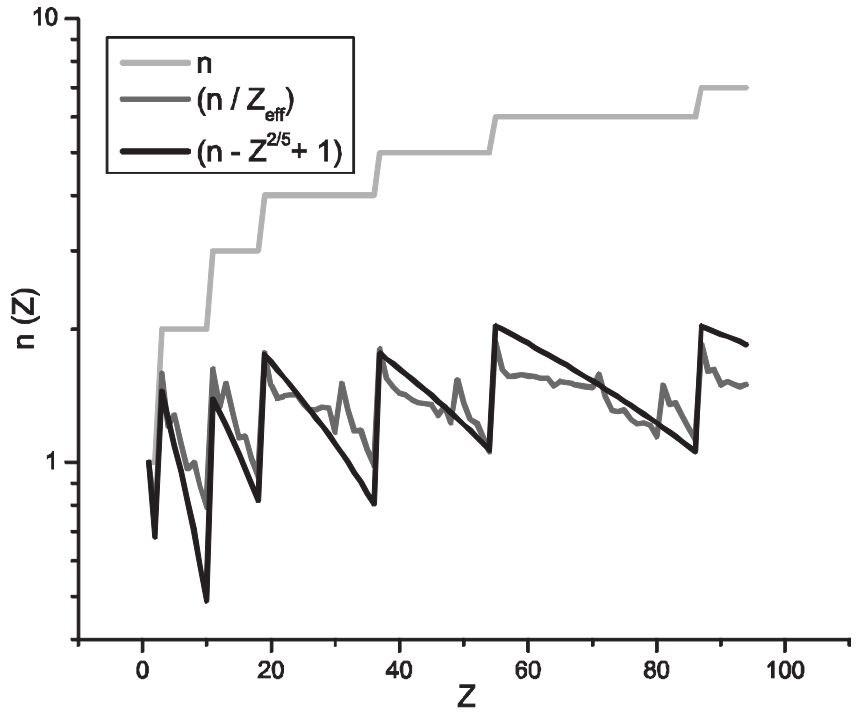

Figure 2S. The $n(Z), n *(Z)=n(Z) / Z_{e f f}(Z)=n(Z)-u(Z)$, and $\bar{n} *(Z)=n(Z)-$ $Z^{2 / 5}+1$ as function of $Z$ for complete $P T$

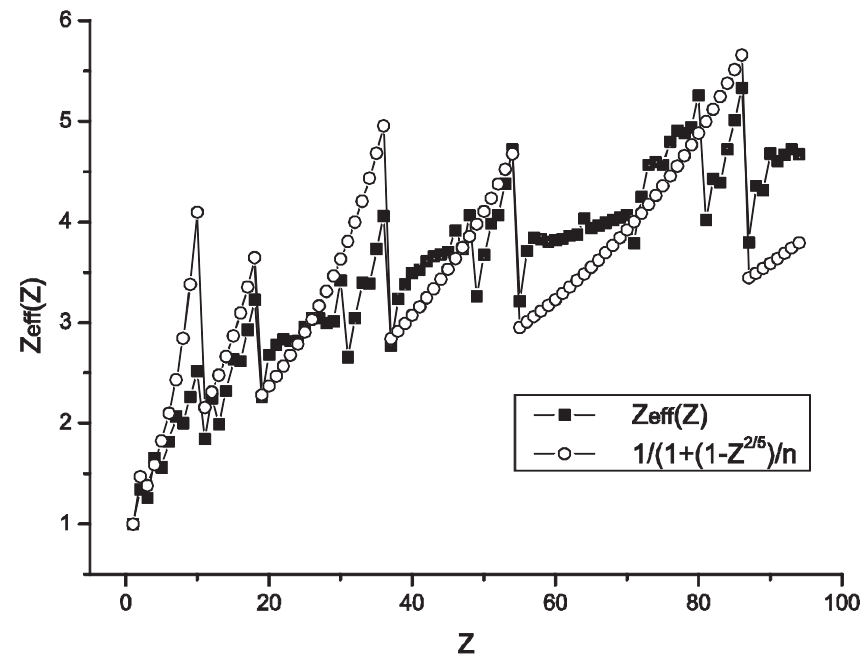

Figure 3S. Experimental $Z_{\text {eff }}($ square $)$ and $\bar{Z}_{\text {eff }}=1 /\left(1+\left(1-Z^{2 / 5}\right) / n(Z)\right)($ circle $)$ as function of $Z$ for complete $P T$ 
Table 1S. Experimental Ionization Potential $(I P)$, Adjusted Ionization Potential $\overline{I P}=13.6 /\left(n(Z)-Z^{2 / 5}+1\right)^{2}$, Experimental Effective Charge $\left(Z_{e f f}\right)$, Adjusted Effective Charge $\bar{Z}_{\text {eff }}=1 /\left(1+\left(1-Z^{2 / 5}\right) / n(Z)\right)$, Experimental Effective Quantum defect $(u)$, and Adjusted Effective Quantum Defect $\bar{u}=Z^{2 / 5}-1$. Data considered in Figures 1 to 5 and $3 \mathrm{~S}$

\begin{tabular}{|c|c|c|c|c|c|c|}
\hline$Z$ & $I P(e V)$ & $\overline{I P}(\mathrm{eV})$ & $\overline{Z_{\text {eff }}}$ & $\overline{\bar{Z}}_{\text {eff }}$ & $u$ & $\bar{u}$ \\
\hline 1 & 13.598 & 13.598 & 1 & 1 & 0 & 0 \\
\hline 2 & 24.587 & 29.365 & 1.34467 & 1.4695 & 0.25632 & 0.31951 \\
\hline 3 & 5.392 & 6.4840 & 1.25941 & 1.3811 & 0.41196 & 0.55185 \\
\hline 4 & 9.322 & 8.5801 & 1.65595 & 1.5887 & 0.79223 & 0.74110 \\
\hline 5 & 8.298 & 11.313 & 1.56235 & 1.8242 & 0.71988 & 0.90365 \\
\hline 6 & 11.26 & 14.994 & 1.81996 & 2.1001 & 0.90107 & 1.0477 \\
\hline 7 & 14.534 & 20.120 & 2.06769 & 2.4328 & 1.03274 & 1.1779 \\
\hline 8 & 13.618 & 27.546 & 2.00147 & 2.8466 & 1.00073 & 1.2974 \\
\hline 9 & 17.422 & 38.830 & 2.26382 & 3.3797 & 1.11654 & 1.4082 \\
\hline 10 & 21.564 & 57.073 & 2.51859 & 4.0974 & 1.20590 & 1.5119 \\
\hline 11 & 5.139 & 7.0329 & 1.84426 & 2.1575 & 1.37333 & 1.6095 \\
\hline 12 & 7.646 & 8.0700 & 2.24958 & 2.3111 & 1.66642 & 1.7019 \\
\hline 13 & 5.986 & 9.2850 & 1.99045 & 2.4790 & 1.49281 & 1.7898 \\
\hline 14 & 8.151 & 10.720 & 2.32268 & 2.6637 & 1.70839 & 1.8738 \\
\hline 15 & 10.486 & 12.432 & 2.63444 & 2.8686 & 1.86124 & 1.9542 \\
\hline 16 & 10.36 & 14.495 & 2.61857 & 3.0974 & 1.85434 & 2.0314 \\
\hline 17 & 12.967 & 17.008 & 2.92957 & 3.3551 & 1.97596 & 2.1058 \\
\hline 18 & 15.759 & 20.109 & 3.22959 & 3.6482 & 2.07109 & 2.1777 \\
\hline 19 & 4.341 & 4.4257 & 2.26005 & 2.2820 & 2.23012 & 2.2471 \\
\hline 20 & 6.113 & 4.7862 & 2.68194 & 2.3731 & 2.50854 & 2.3144 \\
\hline 21 & 7.00 & 5.1799 & 2.77848 & 2.4688 & 2.54143 & 2.3798 \\
\hline 22 & 6.828 & 5.6110 & 2.83445 & 2.5695 & 2.58879 & 2.4432 \\
\hline 23 & 6.746 & 6.0842 & 2.81738 & 2.6756 & 2.58024 & 2.5050 \\
\hline 24 & 6.766 & 6.6053 & 2.82155 & 2.7879 & 2.58234 & 2.5652 \\
\hline 25 & 7.434 & 7.1808 & 2.95756 & 2.9068 & 2.64753 & 2.6239 \\
\hline 26 & 7.902 & 7.8184 & 3.04924 & 3.0331 & 2.68820 & 2.6812 \\
\hline 27 & 7.881 & 8.5271 & 3.04518 & 3.1676 & 2.68645 & 2.7372 \\
\hline 28 & 7.64 & 9.3177 & 2.99826 & 3.3111 & 2.66589 & 2.7920 \\
\hline 29 & 7.726 & 10.203 & 3.01509 & 3.4649 & 2.67334 & 2.8456 \\
\hline 30 & 9.939 & 11.198 & 3.41975 & 3.6300 & 2.83032 & 2.8981 \\
\hline 31 & 5.999 & 12.323 & 2.65682 & 3.8078 & 2.49444 & 2.9495 \\
\hline 32 & 7.899 & 13.598 & 3.04866 & 4.0000 & 2.68795 & 3.0000 \\
\hline 33 & 9.81 & 15.052 & 3.39748 & 4.2085 & 2.82266 & 3.0495 \\
\hline 34 & 9.752 & 16.720 & 3.38742 & 4.4355 & 2.81916 & 3.0982 \\
\hline 35 & 11.841 & 18.644 & 3.73264 & 4.6837 & 2.92837 & 3.1460 \\
\hline 36 & 13.999 & 20.878 & 4.05855 & 4.9564 & 3.01443 & 3.1930 \\
\hline 37 & 4.177 & 4.3857 & 2.77118 & 2.8396 & 3.19571 & 3.2392 \\
\hline 38 & 5.695 & 4.6213 & 3.23578 & 2.9148 & 3.45478 & 3.2846 \\
\hline 39 & 6.217 & 4.8722 & 3.38083 & 2.9929 & 3.52107 & 3.3294 \\
\hline 40 & 6.634 & 5.1397 & 3.49237 & 3.0740 & 3.56831 & 3.3734 \\
\hline 41 & 6.759 & 5.4254 & 3.52512 & 3.1583 & 3.58161 & 3.4169 \\
\hline 42 & 7.092 & 5.7310 & 3.61091 & 3.2460 & 3.61531 & 3.4596 \\
\hline 43 & 7.28 & 6.0582 & 3.65846 & 3.3374 & 3.63330 & 3.5018 \\
\hline 44 & 7.36 & 6.4091 & 3.67851 & 3.4327 & 3.64075 & 3.5434 \\
\hline 45 & 7.459 & 6.7859 & 3.70316 & 3.5321 & 3.6498 & 3.5844 \\
\hline 46 & 8.337 & 7.1914 & 3.91505 & 3.6361 & 3.72288 & 3.6249 \\
\hline 47 & 7.576 & 7.6283 & 3.73209 & 3.7449 & 3.66027 & 3.6649 \\
\hline 48 & 8.994 & 8.0998 & 4.06639 & 3.8590 & 3.77041 & 3.7043 \\
\hline 49 & 5.786 & 8.6098 & 3.26153 & 3.9786 & 3.46698 & 3.7433 \\
\hline 50 & 7.344 & 9.1624 & 3.6745 & 4.1043 & 3.63927 & 3.7818 \\
\hline 51 & 8.641 & 9.7624 & 3.98579 & 4.2365 & 3.74554 & 3.8198 \\
\hline 52 & 9.009 & 10.415 & 4.06978 & 4.3759 & 3.77143 & 3.8574 \\
\hline 53 & 10.451 & 11.127 & 4.3834 & 4.5229 & 3.85933 & 3.8945 \\
\hline 54 & 12.13 & 11.905 & 4.7224 & 4.6784 & 3.94122 & 3.9313 \\
\hline 55 & 3.894 & 3.2919 & 3.21079 & 2.9522 & 4.1313 & 3.9676 \\
\hline 56 & 5.212 & 3.4115 & 3.71463 & 3.0053 & 4.38477 & 4.0035 \\
\hline 57 & 5.577 & 3.5363 & 3.8425 & 3.0598 & 4.43852 & 4.0391 \\
\hline 58 & 5.539 & 3.6667 & 3.82939 & 3.1157 & 4.43317 & 4.0742 \\
\hline 59 & 5.464 & 3.8029 & 3.80337 & 3.1730 & 4.42245 & 4.1091 \\
\hline 60 & 5.525 & 3.9454 & 3.82455 & 3.2319 & 4.43119 & 4.1435 \\
\hline
\end{tabular}


Table 1S. continuation

\begin{tabular}{|c|c|c|c|c|c|c|}
\hline$Z$ & $I P(e V)$ & $\overline{I P}(\mathrm{eV})$ & $Z_{\text {eff }}$ & $\bar{Z}_{\text {eff }}$ & $u$ & $\bar{u}$ \\
\hline 61 & 5.55 & 4.0946 & 3.83319 & 3.2924 & 4.43472 & 4.1776 \\
\hline 62 & 5.644 & 4.2507 & 3.86551 & 3.3546 & 4.44781 & 4.2114 \\
\hline 63 & 5.67 & 4.4143 & 3.87441 & 3.4186 & 4.45138 & 4.2449 \\
\hline 64 & 6.15 & 4.5859 & 4.03507 & 3.4844 & 4.51304 & 4.2780 \\
\hline 65 & 5.864 & 4.7659 & 3.94013 & 3.5521 & 4.47721 & 4.3109 \\
\hline 66 & 5.939 & 4.9550 & 3.96525 & 3.6219 & 4.48685 & 4.3434 \\
\hline 67 & 6.022 & 5.1536 & 3.99286 & 3.6938 & 4.49732 & 4.3756 \\
\hline 68 & 6.108 & 5.3625 & 4.02127 & 3.7679 & 4.50793 & 4.4076 \\
\hline 69 & 6.184 & 5.5823 & 4.04621 & 3.8443 & 4.51713 & 4.4393 \\
\hline 70 & 6.254 & 5.8138 & 4.06905 & 3.9232 & 4.52545 & 4.4706 \\
\hline 71 & 5.426 & 6.0579 & 3.79013 & 4.0048 & 4.41694 & 4.5018 \\
\hline 72 & 6.825 & 6.3155 & 4.25075 & 4.0890 & 4.58848 & 4.5326 \\
\hline 73 & 7.89 & 6.5874 & 4.57038 & 4.1761 & 4.6872 & 4.5633 \\
\hline 74 & 7.98 & 6.8749 & 4.59637 & 4.2663 & 4.69462 & 4.5936 \\
\hline 75 & 7.88 & 7.1791 & 4.56748 & 4.3596 & 4.68637 & 4.6237 \\
\hline 76 & 8.7 & 7.5012 & 4.79925 & 4.4563 & 4.7498 & 4.6536 \\
\hline 77 & 9.1 & 7.8427 & 4.90834 & 4.5567 & 4.77759 & 4.6832 \\
\hline 78 & 9 & 8.2051 & 4.88129 & 4.6608 & 4.77082 & 4.7126 \\
\hline 79 & 9.226 & 8.5902 & 4.9422 & 4.7689 & 4.78597 & 4.7418 \\
\hline 80 & 10.438 & 8.9997 & 5.25681 & 4.8812 & 4.85862 & 4.7708 \\
\hline 81 & 6.108 & 9.4359 & 4.02127 & 4.9981 & 4.50793 & 4.7996 \\
\hline 82 & 7.416 & 9.9010 & 4.43097 & 5.1198 & 4.64589 & 4.8281 \\
\hline 83 & 7.289 & 10.398 & 4.39286 & 5.2466 & 4.63415 & 4.8564 \\
\hline 84 & 8.42 & 10.928 & 4.72139 & 5.3789 & 4.72919 & 4.8845 \\
\hline 85 & 9.5 & 11.497 & 5.01505 & 5.5170 & 4.8036 & 4.9124 \\
\hline 86 & 10.748 & 12.106 & 5.3343 & 5.6613 & 4.8752 & 4.9402 \\
\hline 87 & 4 & 3.2923 & 3.79656 & 3.4444 & 5.15623 & 4.9677 \\
\hline 88 & 5.279 & 3.3828 & 4.3615 & 3.4914 & 5.39505 & 4.9950 \\
\hline 89 & 5.172 & 3.4763 & 4.31708 & 3.5393 & 5.37853 & 5.0222 \\
\hline 90 & 6.084 & 3.5731 & 4.68226 & 3.5883 & 5.50499 & 5.0492 \\
\hline 91 & 5.887 & 3.6733 & 4.60583 & 3.6382 & 5.48019 & 5.0760 \\
\hline 92 & 6.1941 & 3.7771 & 4.67031 & 3.6893 & 5.50117 & 5.1026 \\
\hline 93 & 6.2657 & 3.8846 & 4.72211 & 3.7414 & 5.51761 & 5.1290 \\
\hline 94 & 6.063 & 3.9961 & 4.67417 & 3.7947 & 5.50241 & 5.1553 \\
\hline
\end{tabular}

Table 2S. Experimental Effective Charge $\left(Z_{e f f}\right)$, Slater Effective Charge $\left(Z_{e f f}\right)$, and Adjusted Effective Charge $\bar{Z}_{\text {eff }}=1 /\left(1+\left(1-Z^{2 / 5}\right) / n(Z)\right)$ and theirs $I P(e V)$ for some $Z$. Data considered in Figure 6

\begin{tabular}{|c|c|c|c|c|c|c|}
\hline$Z$ & $Z_{\text {eff }}$ & $I P$ & $Z_{e f f}^{*}$ & $I P^{*}$ & $\bar{Z}_{e f f}$ & $\overline{I P}$ \\
\hline 1 & 1 & 13.598 & 1 & 13.598 & 1 & 13.598 \\
\hline 3 & 1.2594 & 5.392 & 1.3 & 5.75 & 1.381 & 6.4840 \\
\hline 4 & 1.6560 & 9.322 & 1.95 & 12.93 & 1.589 & 8.5801 \\
\hline 5 & 1.5624 & 8.298 & 2.6 & 23.0 & 1.824 & 11.313 \\
\hline 6 & 1.8200 & 11.26 & 3.25 & 35.91 & 2.100 & 14.994 \\
\hline 7 & 2.0677 & 14.534 & 3.9 & 51.7 & 2.433 & 20.120 \\
\hline 8 & 2.0015 & 13.618 & 4.55 & 70.38 & 2.847 & 27.546 \\
\hline 9 & 2.2638 & 17.422 & 5.2 & 91.9 & 3.380 & 38.830 \\
\hline 10 & 2.5186 & 21.564 & 5.85 & 116.3 & 4.097 & 57.073 \\
\hline 11 & 1.8443 & 5.139 & 2.2 & 7.31 & 2.157 & 7.0329 \\
\hline 19 & 2.2601 & 4.341 & 2.2 & 4.11 & 2.281 & 4.4257 \\
\hline 37 & 2.7712 & 4.177 & 2.2 & 2.63 & 2.839 & 4.3857 \\
\hline 55 & 3.2108 & 3.894 & 2.2 & 1.83 & 2.952 & 3.2919 \\
\hline
\end{tabular}

